\title{
First-in-Humans Trial of Dasatinib-Derivative Tracer for Tumor Kinase-Targeted PET
}

\author{
Simone Krebs ${ }^{1}$, Darren R. Veach ${ }^{1,2}$, Lukas M. Carter ${ }^{1}$, Milan Grkovski ${ }^{3}$, Monica Fornier ${ }^{4,5}$, Michael J. Mauro ${ }^{4,5}$, \\ Martin H. Voss ${ }^{4,5}$, Daniel C. Danila ${ }^{4,5}$, Eva Burnazi ${ }^{1,6}$, Manda Null ${ }^{1}$, Kevin Staton ${ }^{1,6}$, Christina Pressl ${ }^{7}$, \\ Bradley J. Beattie ${ }^{3}$, Pat Zanzonico ${ }^{3}$, Wolfgang A. Weber ${ }^{1,2,8}$, Serge K. Lyashchenko ${ }^{1,2,6}$, Jason S. Lewis ${ }^{1,2,6,9}$, \\ Steven M. Larson ${ }^{1,2,9}$, and Mark P.S. Dunphy ${ }^{1,2}$
}

${ }^{1}$ Department of Radiology, Memorial Sloan Kettering Cancer Center, New York, New York; ${ }^{2}$ Department of Radiology, Weill Cornell Medicine, New York, New York; ${ }^{3}$ Department of Medical Physics, Memorial Sloan Kettering Cancer Center, New York, New York; ${ }^{4}$ Department of Medicine, Memorial Sloan Kettering Cancer Center, New York, New York; ${ }^{5}$ Department of Medicine, Weill Cornell Medicine, New York, New York; ${ }^{6}$ Radiochemistry and Molecular Imaging Probes Core, Memorial Sloan Kettering Cancer Center, New York, New York; ${ }^{7}$ Laboratory of Neural Systems, Rockefeller University, New York, New York; ${ }^{8}$ Department of Nuclear Medicine, Technical University of Munich, Munich, Germany; and ${ }^{9}$ Molecular Pharmacology Program, Sloan Kettering Institute, New York, New York

We developed a first-of-kind dasatinib-derivative imaging agent, ${ }^{18} \mathrm{~F}-\mathrm{SKI}-249380\left({ }^{18} \mathrm{~F}-\mathrm{SKI}\right)$, and validated its use for noninvasive in vivo tyrosine kinase-targeted tumor detection in preclinical models. In this study, we assessed the feasibility of using ${ }^{18} \mathrm{~F}-\mathrm{SKI}$ for PET imaging in patients with malignancies. Methods: Five patients with a prior diagnosis of breast cancer, renal cell cancer, or leukemia underwent whole-body PET/CT imaging $90 \mathrm{~min}$ after injection of ${ }^{18} \mathrm{~F}-\mathrm{SKI}$ (mean, $241.24 \pm 116.36 \mathrm{MBq}$ ) as part of a prospective study. In addition, patients underwent either a 30-min dynamic scan of the upper abdomen including, at least partly, cardiac left ventricle, liver, spleen, and kidney $(n=2)$ or three 10-min whole-body PET/CT scans $(n=3)$ immediately after injection and blood-based radioactivity measurements to determine the time course of tracer distribution and facilitate radiation dose estimates. A subset of 3 patients had a delayed whole-body PET/CT scan at $180 \mathrm{~min}$. Biodistribution, dosimetry, and tumor uptake were quantified. Absorbed doses were calculated using OLINDA/EXM 1.0. Results: No adverse events occurred after injection of ${ }^{18} \mathrm{~F}-\mathrm{SKI}$. In total, 27 tumor lesions were analyzed, with a median SUV peak of 1.4 (range, 0.7-2.3) and tumor-to-blood ratios of 1.6 (range, 0.8-2.5) at $90 \mathrm{~min}$ after injection. The intratumoral drug concentrations calculated for 4 reference lesions ranged from 0.03 to $0.07 \mathrm{nM}$. In all reference lesions, constant tracer accumulation was observed between 30 and 90 min after injection. A blood radioassay indicated that radiotracer clearance from blood and plasma was initially rapid (blood half-time, $1.31 \pm 0.81 \mathrm{~min}$; plasma, $1.07 \pm 0.66 \mathrm{~min} ; n=4$ ), followed variably by either a prolonged terminal phase (blood half-time, $285 \pm$ $148.49 \mathrm{~min}$; plasma, $240 \pm 84.85 \mathrm{~min} ; n=2$ ) or a small rise to a plateau $(n=2)$. Like dasatinib, ${ }^{18} \mathrm{~F}-\mathrm{SKI}$ underwent extensive metabolism after administration, as evidenced by metabolite analysis. Radioactivity was predominantly cleared via the hepatobiliary route. The highest absorbed dose estimates (mGy/MBq) in normal tissues were to the right colon $(0.167 \pm 0.04)$ and small intestine $(0.153 \pm 0.03)$. The effective dose was $0.0258 \mathrm{mSv} / \mathrm{MBq}$ (SD, $0.0034 \mathrm{mSv} / \mathrm{MBq})$. Conclusion: ${ }^{18} \mathrm{~F}-\mathrm{SKI}$ demonstrated significant tumor uptake,

Received Aug. 8, 2019; revision accepted Mar. 5, 2020.

For correspondence or reprints contact: Mark P.S. Dunphy, Memorial Sloan Kettering Cancer Center, 1275 York Ave., Room S113E, New York, NY 10065 .

E-mail: dunphym@mskcc.org

Published online Mar. 13, 2020.

COPYRIGHT (C 2020 by the Society of Nuclear Medicine and Molecular Imaging. distinct image contrast despite low injected doses, and rapid clearance from blood.

Key Words: ${ }^{18} \mathrm{~F}-\mathrm{SKI}-249380$; dasatinib; PET imaging; Src kinase

J Nucl Med 2020; 61:1580-1587

DOI: 10.2967/jnumed.119.234864

\section{A}

hallmark of cancer relies on the deregulation of cell signaling pathways, providing a rationale for mechanism-based targeted therapies $(1,2)$. The Src family kinases regulate tumor cell proliferation, invasion, angiogenesis, and metastasis $(3,4)$. Src family kinase activity is upregulated in various malignancies, making the Src family a promising target for tumor therapy (5). Dasatinib is an orally bioavailable small-molecule inhibitor of Src family kinases (and other tyrosine kinases) that has been approved for the treatment of chronic myelogenous leukemia (6). It is being extensively studied in both preclinical and clinical studies for the management of solid malignancies, including breast cancer (7), renal cell cancer (8), non-small cell lung cancer, prostate cancer, and glioma (Fig. 1) (9,10).

So far, dasatinib has been evaluated both as a single agent and as a combination therapy in solid tumors, with low RECIST response rates (10). However, Demetri et al. reported a phase 1 study investigating dasatinib in patients with metastatic solid tumors refractory to standard therapies or for which no effective standard therapy exists (11). Although no objective tumor responses were seen, durable stable disease was observed in $16 \%$ of patients. Thus, there is an obvious need for patient selection that can benefit from dasatinib-based treatment and monitoring of therapeutic outcome.

PET enables noninvasive assessment of target expression, monitoring response to tumor treatments, and outcome prediction. An emerging promising approach for personalized therapy is the use of PET for monitoring radioactively labeled drugs in vivo. PETbased monitoring of the pharmacokinetic behavior of radiolabeled drugs can also provide a noninvasive, quantitative read-out of the 


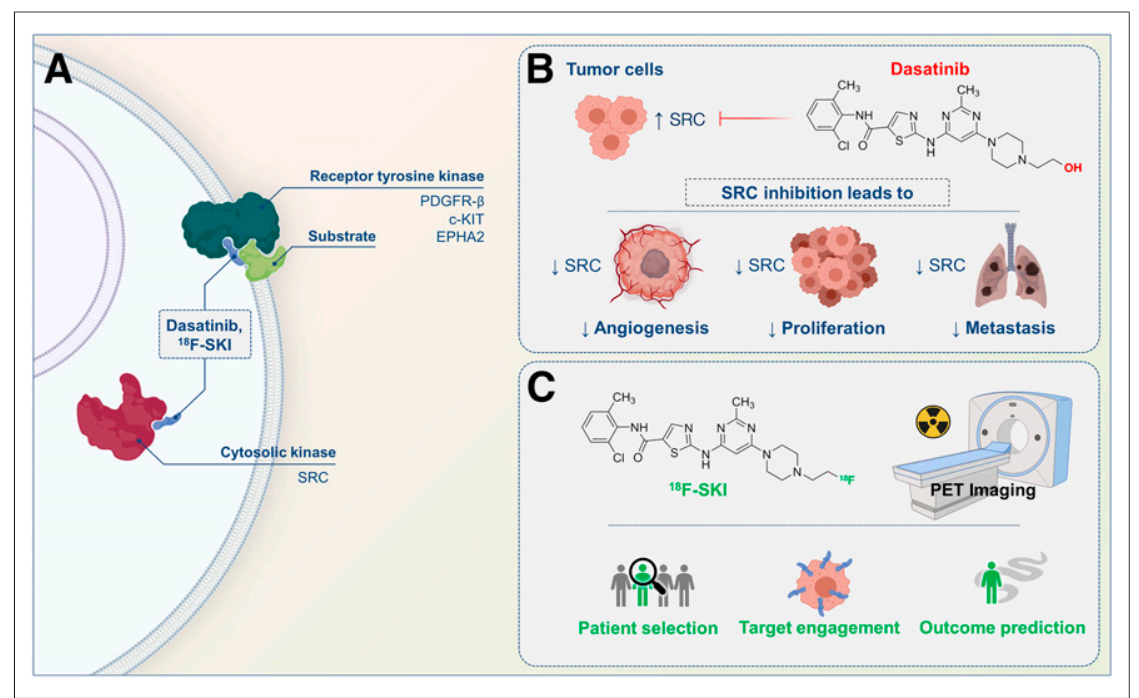

FIGURE 1. Scheme of dasatinib for tumor treatment and radiolabeled dasatinib-derivative ${ }^{18} \mathrm{~F}$ SKI for PET imaging. (A) Cytosolic and membrane targets of dasatinib and ${ }^{18} \mathrm{~F}-\mathrm{SKI}$. (B) Dasatinib inhibits SRC signaling, which plays central role in neoplastic disorders and leads to other biologic actions. (C) ${ }^{18} \mathrm{~F}-\mathrm{SKI}$-based PET imaging enables visualization of target engagement in patients with variety of tumors and is thus potential pharmacometric biomarker to guide dasatinib-based cancer therapies.

target engagement of radiolabeled drugs at the tumor site, which possibly correlates with its sensitivity or resistance to therapy.

Prior reports have indicated the feasibility of tyrosine kinasebased PET imaging $(12,13)$. However, our group was the first to report on a ${ }^{18} \mathrm{~F}$-radiolabeled dasatinib derivative, ${ }^{18} \mathrm{~F}-\mathrm{SKI}-249380$ $\left({ }^{18} \mathrm{~F}-\mathrm{N}\right.$-(2-chloro-6-methylphenyl)-2-(6-(4-(2-fluoroethyl)piperazin-1-yl)-2-methylpyrimidin-4-ylamino)thiazole-5-carboxamide $\left.\left[{ }^{18} \mathrm{~F}-\mathrm{SKI}\right]\right)(14,15)$. Using in vitro kinase and cellular assays, we investigated its kinase inhibition profile and verified that it closely parallels that of dasatinib. ${ }^{18} \mathrm{~F}$-SKI demonstrated equipotent inhibition of proliferation of Bcr-Abl-dependent cells K562 and $\mathrm{M} 07 \mathrm{e} / \mathrm{p} 210^{\mathrm{bcr}-\mathrm{abl}}$ at $0.10 \mathrm{nM}$. To further substantiate the similarities of dasatinib and ${ }^{18} \mathrm{~F}-\mathrm{SKI}$, we examined the kinase inhibition of ${ }^{18} \mathrm{~F}-\mathrm{SKI}$ at $10 \mathrm{nM}$ in a panel of 21 kinases, including $\mathrm{Abl}$, Src, Kit, EphA2, EphB4, and PDFR $\alpha$. Using mouse models bearing K562 tumor xenografts, we verified selective in vivo targeting. ${ }^{18} \mathrm{~F}$-SKI PET enabled noninvasive assessment of dasatinib-derivative target engagement in all 5 mice. Furthermore, our preclinical data demonstrated favorable dosimetry, supporting the rationale for clinical application.

The purpose of this study was to assess the utility of ${ }^{18} \mathrm{~F}-\mathrm{SKI}$ for PET imaging in a first-in-humans pilot study and investigate detailed biodistribution and radiation dosimetry. Five patients with metastatic breast cancer (MBC), renal cell cancer, and leukemia were enrolled.

\section{MATERIALS AND METHODS}

\section{Clinical Study with ${ }^{18} \mathrm{~F}-\mathrm{SK}$}

The study was an open-label, nonrandomized, first-in-humans microdose pilot trial conducted at Memorial Sloan Kettering Cancer Center with the approval of the institutional review board (ClinicalTrials.gov NCT01916135) and under the auspices of an Investigational New Drug application acknowledged by the Food and Drug Administration. All participants provided written informed consent.

\section{Patient Selection}

Eligibility criteria included a minimum age of $21 \mathrm{y}$, serum renal and hepatic function test values less than 1.5 - to 2.5 -fold greater than the lab-specific upper limit of normal, and a histologically confirmed solid, myeloid, or lymphoid neoplasm. The disease was either radiologically measurable, detectable by biopsy (e.g., bone marrow), or confirmed by peripheral blood assays obtained within 6 wk of study enrollment. Serum complete blood count and liver and renal function tests were obtained within 2 wk before study participation. Exclusion criteria included pregnancy, breastfeeding, and acute major illness. No patients were taking dasatinib or other medications known as tyrosine kinase inhibitors or inducers of cytochrome $\mathrm{P} 450$ isoenzyme CYP3A4 (because dasatinib metabolism is CYP3A4-dependent) for less than 2 wk before participation (16).

\section{Radiopharmaceutical Preparation and Administration}

Manufacture of clinical-grade ${ }^{18} \mathrm{~F}-\mathrm{SKI}$ and subsequent dose preparation were performed by the Radiochemistry and Molecular Imaging Probes Core at Memorial Sloan Kettering. The synthesis has been described previously (Supplemental Fig. 1; supplemental materials are available at http://jnm.snmjournals.org) (17).

The synthesis of ${ }^{18} \mathrm{~F}$-SKI involved ${ }^{18} \mathrm{~F}$-fluoride nucleophilic incorporation onto the precursor molecule, followed by drug substance isolation by preparatory high-performance liquid chromatography (HPLC) purification (Supplemental Fig. 1). Briefly, to obtain the ${ }^{18} \mathrm{~F}$ in the appropriate form, cyclotron-produced ${ }^{18} \mathrm{~F}$ was trapped on the quaternary methyl ammonium Sep-Pak (Waters), eluted with $0.6 \mathrm{~mL}$ of tetrabutylammonium bicarbonate $(0.075 \mathrm{M})\left(\mathrm{N}(n-\mathrm{Bu})_{4} \mathrm{HCO}_{3}\right)$ into a reaction vial containing a Teflon (Chemours) magnetic stir bar, and dried by being heated at $90^{\circ} \mathrm{C}$ under vacuum. The precursor, $2 \mathrm{mg}$ of the $\mathrm{N}$-(2-chloro-6-methylphenyl)-2-((6-(4-(2-chloroethyl) piperazin-1-yl)-2-methylpyrimidin-4-yl)amino)thiazole-5-carboxamide (Advanced Biomedical Compounds), dissolved in $0.1 \mathrm{~mL}$ of dimethyl sulfoxide and $0.3 \mathrm{~mL}$ of acetonitrile, was then added to the reaction vessel and the reaction was heated at $80^{\circ} \mathrm{C}$ for $50 \mathrm{~min}$. Once the fluorination reaction was complete, the unreacted ${ }^{18} \mathrm{~F}$-tetrabutylammonium fluoride was removed via hydrophilic-lipophilic balance solidphase extraction purification. The drug substance, ${ }^{18} \mathrm{~F}-\mathrm{SKI}$, was isolated by preparatory HPLC, with a gradient of $20 \mathrm{mM}$ sodium acetate and $85 \%$ acetonitrile solution as the mobile phase (Phenomenex) and a Gemini C18, pentafluorophenyl, $5 \mu \mathrm{m}, 250 \times 10 \mathrm{~mm}$ column as the stationary phase; a flow of $4.0 \mathrm{~mL} / \mathrm{min}$; and an ultraviolet detector wavelength of $320 \mathrm{~nm}$. The HPLC system was a Shimadzu Prominence 20 series, equipped with an ultraviolet detector and a Flow-Ram sodium iodide radioactivity detector supplied by Lablogic. The HPLC eluate fraction containing the drug substance, the radioactive peak for which was eluting at $18.5 \pm 3 \mathrm{~min}$, was collected into a pear-shaped glass flask containing $40 \mathrm{~mL}$ of sterile water for injection (of U.S. Pharmacopeia grade). To remove the HPLC mobile-phase solvents, the reaction mixture was loaded onto a light $\mathrm{C} 18$ cartridge and washed with an additional $10 \mathrm{~mL}$ of sterile water. The drug substance was eluted from the C18 Sep-Pak with $0.5 \mathrm{~mL}$ of ethanol, followed by $9.5 \mathrm{~mL}$ of normal saline, through a $0.22-\mu \mathrm{m}$ sterilizing filter into a sterile, apyrogenic, U.S. Pharmacopeia type I glass vial, sealed with a rubber septum, and crimped with an aluminum crimp. 
The final drug product underwent quality control testing before batch release for patient administration in accordance with acceptance specifications for radiochemical purity, endotoxin content, sterilizing filter integrity, $\mathrm{pH}$, appearance, and radionuclide identity confirmation. Sterility was tested after release.

The radiochemical purity and specific activity measurements of ${ }^{18} \mathrm{~F}$-SKI were performed using a Shimadzu Prominence HPLC system equipped with a $20-\mu \mathrm{L}$ injection loop, an SPD-20A ultraviolet-visible-light detector, and an LC-20AT mobile-phase pump. The stationary phase was a Phenomenex Kinetex C-18, 5- $\mu \mathrm{m}, 250 \times 4.6 \mathrm{~mm}$ column. An isocratic mixture of $50 \%$ acetonitrile in $20 \mathrm{mM}$ sodium acetate buffer at a flow rate of $1 \mathrm{~mL} / \mathrm{min}$ was used as the mobile phase. A Flow-RAM radioactivity detector (Lablogic) was used to detect radioactive compounds. The mass of ${ }^{18} \mathrm{~F}-\mathrm{SKI}$ in the final drug product was calculated by comparing the area under the curve for the corresponding ultraviolet peak against the mass standard curve of known concentrations of ${ }^{19} \mathrm{~F}-\mathrm{SKI}-249380$ at $320 \mathrm{~nm}$. The specific activity was calculated by dividing the total radioactivity at the end of synthesis by the determined mass of ${ }^{18} \mathrm{~F}-\mathrm{SKI}$ in the final drug product.

${ }^{18} \mathrm{~F}-\mathrm{SKI}$ was administered to subjects at an activity of $241.24 \pm$ 116.36 MBq (range, 114.7-421.1 MBq) by intravenous bolus injection. The administered activity for the clinical trial was based on human dosimetry estimates extrapolated from our published murine dosimetry (15).

\section{Toxicity Monitoring}

Preinjection and postscan vital signs (heart rate, blood pressure, and body temperature) were taken, and clinical symptoms were monitored. Physical examinations were performed immediately after injection, and 2 catheters (for tracer administration and blood sampling) were accessed to separate veins in the upper extremities. Patients voided the urinary bladder immediately before ${ }^{18} \mathrm{~F}-\mathrm{SKI}$ injection and were monitored for signs and symptoms up to $4 \mathrm{~h}$ after injection. Patients were contacted about $24 \mathrm{~h}$ after injection for further inquiry and were advised to contact the study team if they experienced any signs or symptoms in the $30 \mathrm{~d}$ after injection. Clinical toxicity was monitored up to 1 wk after injection using the Common Terminology Criteria for Adverse Events, version 4.0.

\section{PET/CT Imaging with ${ }^{18} \mathrm{~F}-\mathrm{SKI}$}

All 5 patients underwent whole-body (skull to proximal thigh) PET/CT imaging (GE Healthcare PET/CT Discovery STE and 710 scanners with time-of-flight capability) in 3-dimensional mode at $90 \mathrm{~min}$ after injection. Patients were asked to void their bladders before imaging.

A low-dose CT scan $(120 \mathrm{kVp}, 80-\mathrm{mA}$ tube current, helical pitch of 1.5:1, estimated radiation dose of $9.0 \mathrm{mGy}$ ) was acquired before PET imaging for attenuation correction and anatomic localization. The total acquisition time was 18 or $21 \min$ for 6 or 7 bed positions, respectively ( $3 \mathrm{~min}$ per position). Images were reconstructed using a 700 -mm field of view into a $128 \times 128$ matrix with iterative ordered-subset expectation maximization (16 subsets; 2 iterations) with a 6.4-mm 2-dimensional Gaussian filter and heavy axial 3-point smoothing ([1 21 1 $1 / 4)$. Manufacturers' corrections were used, including CT-based attenuation, scatter, and SharpIR (point-spread-function correction). PET scanner quality assurance, including an ${ }^{18} \mathrm{~F}$ well counter calibration, was performed quarterly per American College of Radiology guidelines.

All patients underwent additional imaging after injection to determine the tracer distribution time course and facilitate radiation dose estimates: 10-min whole-body sweeps at 0-10 min, 10-20 min, and 20-30 $\min (n=3)$ or 30-min dynamic scanning of the upper abdomen, including, at least partly, cardiac left ventricle, liver, and spleen $(n=2)$. Three of the 5 patients underwent an additional delayed whole-body PET/CT scan at 180 min (Supplemental Fig. 2).

\section{Image Interpretation, Lesion Detection, and Data Analysis}

The whole-body static PET/CT scans acquired at $90 \mathrm{~min}$ after injection were first interpreted without reference to other imaging modalities. Lesions with focal radiotracer uptake and retention greater than blood-pool activity were interpreted as metastatic disease.

Volumes of interest (VOIs) were generated over lesions using a Hermes imaging workstation (GOLD, version 4.4-B; Hermes Medical Solutions) and AW Suite on Centricity PACS (GE Healthcare Integrated IT Solutions). Typically, a value of $40 \%-50 \%$ of maximum tracer uptake was used as a threshold, but this was guided by interpretation of the CT scan. In total, 27 lesions were analyzed.

For normal tissues (brain, left cardiac ventricle wall, lungs, liver, spleen, pancreas, kidneys, small intestines, spine, humerus, and skeletal musculature), regions of interest were drawn over at least 5 consecutive transaxial PET slices and combined to generate VOIs. Tracer uptake was quantified by SUVs normalized to body weight. For lesion VOI, SUV $\mathrm{Sean}_{\text {men }} \mathrm{SUV}_{\max }$ (maximum voxel in the VOI), and $S_{U V} V_{\text {peak }}\left(\mathrm{SUV}_{\text {mean }}\right.$ of the hottest $1 \mathrm{~cm}^{3}$ in the VOI) were recorded. For normal tissues, $\mathrm{SUV}_{\text {mean }}$ and $\mathrm{SUV}_{\text {peak }}$ were used. Tumor-to-blood SUV ratios, defined as $\mathrm{SUV}_{\text {peak }}$ (lesion)/SUV $/ \mathrm{S}_{\text {mean }}$ (left cardiac ventricle), were also calculated. Intratumoral ${ }^{18} \mathrm{~F}$-SKI mass concentrations were calculated using the specific molar activity at the time of injection and $\mathrm{SUV}_{\text {mean }}$ (lesion).

$\mathrm{SUV}_{\text {mean }}$ (lesion) is defined as the ratio of mean lesional radioactivity concentration $\left(\mathrm{MBq} / \mathrm{cm}^{3}\right)$ to the whole-body radioactivity concentration (injected dose in MBq/patient mass in g). Using the decay-corrected specific molar activity of ${ }^{18} \mathrm{~F}-\mathrm{SKI}$ expressed in $\mathrm{MBq} / \mathrm{nmol}$, we calculate the number of molecules of ${ }^{18} \mathrm{~F}-\mathrm{SKI}$ in the lesional volume in $\mathrm{cm}^{3}$ as defined during VOI analysis using coregistered PET and CT images. With unit conversion, we obtain the average lesional mass concentration of ${ }^{18} \mathrm{~F}$-SKI expressed as a nanomolar concentration. We make several assumptions in this calculation. We assume that the average density of tissue is $1 \mathrm{~g} / \mathrm{cm}^{3}$. Further, we assume that the tumor radioactivity present is due only to intact radiotracer.

A limitation of this work lies in our assumptions about the molecular species responsible for tumoral uptake, retention, and clearance. It is possible that the tumoral uptake was due, in part, to unanticipated radiolabeled species that confound the use of ${ }^{18} \mathrm{~F}-\mathrm{SKI}$ as a surrogate marker for dasatinib. We posit that the interpretation of the imaging data and quantification of intratumoral drug concentration is valid on the basis of the following discussion.

The translation of the tumor activity concentration into drug concentration is predicated on the assumption that the activity in the tumor is in the form of the administered radiotracer, that is, ${ }^{18} \mathrm{~F}-\mathrm{SKI}$. The direct measurement of the radiochemical composition of the tumor activity is, of course, impractical in a clinical setting and was beyond the scope of this limited imaging trial design. As enumerated below, however, we believe that the collective available evidence, although largely circumstantial, strongly supports this assumption.

First, among the 27 lesions identified in the 5 patients described here, the time-activity data reveal stable activity concentrations (i.e., flat time-activity curves) in the tumors. Specifically, the tumor mean ( $\pm \mathrm{SD}$ ) SUV is $1.4 \pm 0.6$ and $1.4 \pm 0.4$ at 30 and $90 \mathrm{~min}$, respectively, after injection of ${ }^{18} \mathrm{~F}-\mathrm{SKI}$. These fixed SUVs indicate little to no clearance of activity over this 60-min interval. This, in turn, is consistent with high-affinity binding of the ${ }^{18} \mathrm{~F}$-labeled species within the tumor-binding specifically characteristic of the ${ }^{18} \mathrm{~F}-\mathrm{SKI}$ itself but not of any possible non-kinase-binding or low-affinity-binding ${ }^{18} \mathrm{~F}$-labeled metabolites. If significant concentrations of such metabolites were present in tumor, these would be cleared rapidly from tumor and the tumor SUV would therefore sharply decline, which clearly was not observed. 
Second, there is no notable skeletal activity in any of the patients imaged, indicating that little to no free ${ }^{18} \mathrm{~F}$-fluoride was produced in vivo. This, in turn, means 2 things: that there is little to no ${ }^{18} \mathrm{~F}$-fluoride, specifically, in tumor, and that catabolism at the $\mathrm{N}$-ethylpiperazine moiety, if occurring, is not generating free fluoride.

Third, the analysis of plasma-borne activity by HPLC revealed only 3 radioactive species in blood: a rapidly eluting, polar species; a latereluting, somewhat hydrophobic species not yet definitively identified; and an even later-eluting, more hydrophobic species corresponding to the intact ${ }^{18} \mathrm{~F}$-SKI. It is unlikely the polar species significantly contributes to tumor activity, as its highly polar nature would likely prevent entry into and subsequent retention by tumor (and other) cells. The second of these peaks likely corresponds to an ${ }^{18} \mathrm{~F}-\mathrm{SKI}$-derived aryl-hydroxylated species that may bind to the kinase but only with low affinity, as with dasatinib (18). Importantly, if that species were present within tumor cells, its low binding affinity and hydrophobic character would allow its rapid egress from these cells and rapid clearance of the associated activity from the tumor. However, as detailed in the above text, the tumor ${ }^{18} \mathrm{~F}$ activity does not decline at all between 30 and 90 min of ${ }^{18} \mathrm{~F}$-SKI injection, indicating that little to none of this hydroxylated ${ }^{18} \mathrm{~F}-\mathrm{SKI}$ species is actually present in tumor. The only other ${ }^{18} \mathrm{~F}$-labeled species in vivo, based on the HPLC analysis, is ${ }^{18} \mathrm{~F}$-SKI itself; plausibly, therefore, it is mostly ${ }^{18} \mathrm{~F}-\mathrm{SKI}$ actually present in tumor.

Fourth, one may reasonably speculate that, in light of the specific site of the fluorination of the dasatinib molecule, the oxidative and conjugation metabolism to which dasatinib itself is susceptible is blocked at that site. This is because the fluorine replaces a hydroxyl group, which, based on the relevant literature, facilitates some of the metabolic clearance of dasatinib (18). The dearth of ${ }^{18} \mathrm{~F}$-SKI metabolites after ${ }^{18} \mathrm{~F}-\mathrm{SKI}$ injection, as demonstrated by HPLC, is consistent with that speculation. Furthermore, alternate catabolic pathways that destabilize the $\mathrm{C}-\mathrm{F}$ bond, releasing ${ }^{18} \mathrm{~F}$-fluoride, did not appear to be in operation.

The points in the foregoing discussion, we feel, provide a compelling validation of the assumption that the ${ }^{18} \mathrm{~F}$ activity in tumor is entirely or almost entirely intact ${ }^{18} \mathrm{~F}$-SKI.

\section{Blood Radioassays}

Blood samples were collected at 1, 5, 15, 30, 90, and 180 min after tracer injection. Activity in whole blood and plasma samples was radioassayed by a well counter (1480 Wizard 3" automatic $\gamma$-counter; PerkinElmer), and measured activity concentrations were converted to percentage injected activity per liter. Areas under the curve and corresponding residence times were derived by analytic integration.

\section{Absorbed Dose Calculations}

Absorbed doses to total body and individual organs were based on the time-activity data derived from each patient's set of PET/CT images. VOIs were drawn over the liver, spleen, heart contents, bone, kidneys, brain, lungs, salivary glands, and urinary bladder using the Hermes Hybrid Viewer (Hermes Medical Solutions). As is routine, the PET images were parameterized in terms of SUVs, and the mean SUVs in the foregoing organs (i.e., VOIs) were converted to fractions of the administered activity in each organ by scaling by the respective fractional masses, based on the organ masses in the OLINDA/EXM 2.0 software (Hermes Medical Solutions) for the 70-kg reference adult. The resulting time-activity curves (Supplemental Fig. 3) were fit to exponential functions and the fitted functions analytically integrated to infinity to derive the organ time-integrated activity coefficients in $\mathrm{MBq}-\mathrm{h} / \mathrm{MBq}$ (or simply $\mathrm{h}$ ), incorporating the physical decay of ${ }^{18} \mathrm{~F}$. To derive the time-integrated activity coefficient urinary bladder contents, the voiding bladder model in OLINDA/EXM 2.0 was used, with the bladder activity estimated by multiplying the mean
SUV in a VOI encompassing the bladder activity with its fractional mass. Although there was visualizable, but low, activity in the urinary bladder, there was actually no demonstrable total-body clearance of ${ }^{18} \mathrm{~F}$ over the time course of the PET studies. Therefore, a single totalbody biologic clearance half-time was assumed and arbitrarily set to $100 \mathrm{~h}$ to estimate the bladder content time-integrated activity coefficient and its contribution to the bladder-wall absorbed dose; the bladder voiding interval was set to $3 \mathrm{~h}$. Significant uptake was observed in the gut, but not surprisingly, the time course of gut activity was irregular and, again, there was no demonstrable clearance of activity. Therefore, to estimate the respective time-integrated activity coefficients for left colon, small intestine, right colon, and rectum, the International Commission on Radiological Protection human alimentary tract model, as implemented in OLINDA/EXM 2.0 (18), was used. It was assumed that the activity enters the small intestine from the liver, with the fraction of administered activity entering the small intestine (0.23) calculated by taking the maximum value for the liver $\mathrm{SUV}_{\text {mean }}$ and multiplying it by its fractional liver mass and assuming no reabsorption of intestinal activity. The remainder-of-body time-integrated activity coefficient was derived by subtracting the sum of the timeintegrated activity coefficients of the individual organs from that of the total body. The total-body time-integrated activity coefficient was calculated using the total-body time-activity curve for the activity in the PET field of view (from mid thigh to vertex), yielding a fractional administered activity averaging approximately only 0.9 at zero time among the 5 patients (presumably because the entire body was not in the field of view) (Supplemental Fig. 4) and equating the totalbody effective half-life with the physical half-life of ${ }^{18} \mathrm{~F}$. There was no demonstrable total-body clearance over the time course of the PET imaging studies. The 70-kg reference-adult organ absorbed doses were then calculated using the OLINDA/EXM 2.0 software (19). The timeintegrated activity coefficients and absorbed doses are presented in Supplemental Tables 2 and 3, respectively.

\section{Statistics}

Patient data were summarized by descriptive statistics, including mean, SD, coefficients of variation, range, and $95 \%$ confidence intervals, when appropriate.

\section{RESULTS}

\section{${ }^{18}$ F-SKI Administration and Safety}

Five patients with a prior diagnosis of breast cancer, renal cell cancer, or leukemia were administered ${ }^{18} \mathrm{~F}$-SKI, underwent at least 1 whole-body PET/CT scan, and were included in data analysis. Patient characteristics are summarized in Table 1. Both patients with breast cancer had undergone surgery and multiple lines of endocrine therapy and chemotherapy. The patient with renal cell cancer underwent surgery only. Of the 2 patients with leukemia, one was diagnosed with Philadelphia chromosome-positive pre-B acute lymphoblastic leukemia (patient 4) and had evidence of disease, as confirmed by marrow biopsy or blood assay within 6 wk after study enrollment. At the time of the imaging study, however, the patient had no evidence of disease, as confirmed by blood and bone marrow assays. The other patient was diagnosed with chronic myelogenous leukemia (patient 5) with an ongoing myeloid blast crisis evident on blood and bone marrow assays at the time of the PET scan. Both patients were receiving targeted therapy with the tyrosine kinase inhibitor imatinib about 2 mo before ${ }^{18} \mathrm{~F}$-SKI PET. After the scan, both continued to receive treatment with tyrosine kinase inhibitors.

The mean administered activity was $241.24 \pm 116.36 \mathrm{MBq}$ (range, 114.7-421.1 MBq), with a mean radiochemical purity of 
TABLE 1

Demographic and Clinical Characteristics of Patients and Overview of Lesion Analyses

\begin{tabular}{|c|c|c|c|c|c|c|c|c|c|}
\hline Patient & Sex & Age (y) & $\begin{array}{c}\text { Cancer } \\
\text { type }\end{array}$ & Lesion site & $\begin{array}{c}\text { Lesions } \\
\text { analyzed }(n)\end{array}$ & $\begin{array}{l}S_{\text {SUV }} \text { peak } \\
\text { (30 min) }\end{array}$ & $\begin{array}{l}\text { TNR-B } \\
\text { (30 min) }\end{array}$ & $\begin{array}{l}S_{\text {SUV }} \text { peak } \\
\text { (90 min) }\end{array}$ & $\begin{array}{c}\text { TNR-B } \\
\text { (90 min) }\end{array}$ \\
\hline 1 & $F$ & 59 & Breast & Bone & 16 & $1.23 \pm 0.4$ & $2.33 \pm 0.8$ & $1.27 \pm 0.3$ & $1.46 \pm 0.4$ \\
\hline 2 & $\mathrm{~F}$ & 55 & Breast & Bone, uterus & 9 & $1.53 \pm 0.5$ & $2.18 \pm 0.6$ & $1.42 \pm 0.4$ & $1.58 \pm 0.4$ \\
\hline 3 & $M$ & 77 & $\mathrm{RCC}$ & Muscle & 1 & 1.59 & 1.52 & 1.52 & 1.62 \\
\hline $4^{*}$ & $\mathrm{~F}$ & 58 & ALL & $\mathrm{NA}$ & NA & NA & NA & NA & NA \\
\hline 5 & $\mathrm{~F}$ & 59 & $\mathrm{CML}$ & Bone marrow & 1 & 3.23 & 3.32 & 2.12 & 1.89 \\
\hline
\end{tabular}

*Patient with evidence of disease in blood and bone marrow at time of study inclusion but no evidence of disease at time of imaging.

TNR-B: tumor-to-blood ratio; RCC = renal cell cancer; $A L L=$ Philadelphia chromosome-positive pre-B acute lymphoblastic leukemia; $\mathrm{NA}=$ not applicable; $\mathrm{CML}=$ chronic myelogenous leukemia.

$97 \% \pm 2.1 \%$ (range, $94.48 \%-99.33 \%$ ), a specific molar activity of

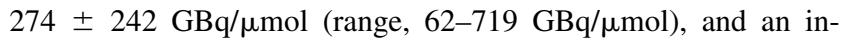
jected mass of $1.7 \pm 1.6 \mu \mathrm{g}$ (range, $0.3-4.1 \mu \mathrm{g}$ [0.6-8.3 nmol]). No infusion-related symptoms were noted. No significant changes were evident in heart rate, blood pressure, or body temperature before versus after injection, and no subsequent adverse events were observed in the follow-up period (mean, $7 \mathrm{~d}$ ).

\section{PET/CT Imaging with ${ }^{18} \mathrm{~F}-\mathrm{SKI}$}

${ }^{18} \mathrm{~F}$-SKI PET/CT revealed positive lesions in 4 of 5 patients. Patient 4 exhibited no disease on PET, as subsequently confirmed by blood and bone marrow assays. On visual inspection, images demonstrated little to no uptake in the brain, pituitary gland, salivary glands, thyroid, blood pool, lung, and musculature (Figs. $2-4)$. The predominant route of ${ }^{18} \mathrm{~F}-\mathrm{SKI}$ excretion was hepatobiliary, and substantial radioactivity in the intestine was observed. Minimal urinary excretion was detected. Quantitative characterization of tumor and normal-organ uptake values based on VOI analysis is provided in Supplemental Figure 3.

Blood radioassay indicated that radiotracer clearance from blood and plasma was initially rapid (blood half-time, $1.31 \pm$ $0.81 \mathrm{~min} ;$ plasma half-time, $1.07 \pm$ $0.66 \mathrm{~min} ; n=4$ ), followed variably by either a prolonged terminal phase (blood half-time, $285 \pm 148.49 \mathrm{~min}$; plasma halftime, $240 \pm 84.85 \mathrm{~min} ; n=2$ ) or a small rise to a plateau $(n=2)$ (Fig. 5). The riseto-plateau pattern possibly indicates systemic recirculation of an isotope extravasated into bodily tissues during the initial phase. The blood-to-plasma radiotracer activity ratio was $1.3 \pm 0.1$ at 5 min versus $1.1 \pm 0.2$ at $30 \mathrm{~min}$. Like dasatinib, ${ }^{18} \mathrm{~F}-\mathrm{SKI}$ undergoes extensive metabolism during the 2-h period after administration (18), but plasma radiotracer was predominantly intact parent compound (Supplemental Table 1). In vivo tracer metabolism yielded no free ${ }^{18} \mathrm{~F}$ on radio-HPLC.

In total, 27 lesions were examined on ${ }^{18} \mathrm{~F}-\mathrm{SKI} \mathrm{PET} / \mathrm{CT}$ (16 in 1 patient, 9 in 1 patient, and 1 in 2 patients) (Table 1). Bone was the most common site of disease, with 24 lesions analyzed in the 2 patients with MBC (Table 1; Figs. 2 and 3). Two additional lesion sites included the uterus (a subsequently biopsy-proven metastasis in patient 2, with MBC) (Fig. 3) and skeletal
FIGURE 2. Patient with breast cancer and osseous metastases (patient 1). (A) Serial axial fused ${ }_{18}^{18}$ F-SKI PET/CT and PET images set at same intensity scale show visualization of osseous metastases. Some lesions are already visualized at early time points (30 min; orange arrows), whereas others are visualized at later time points after injection (180 min; white arrows). (B) SUV $_{\text {peak }}$ time curves illustrate ${ }^{18} \mathrm{~F}-\mathrm{SKI}$ uptake in osseous lesions. (C) Maximum-intensity-projection image at $180 \mathrm{~min}$ shows distribution of ${ }^{18} \mathrm{~F}$-SKI. Scattered osseous metastases involving, for example, skull, ribs, pelvic bones, and bilateral femora are seen. (D) On same day, ${ }^{99 \mathrm{~m} T c-m e t h-}$ ylene diphosphonate bone scan shows osseous metastases in similar distribution. 


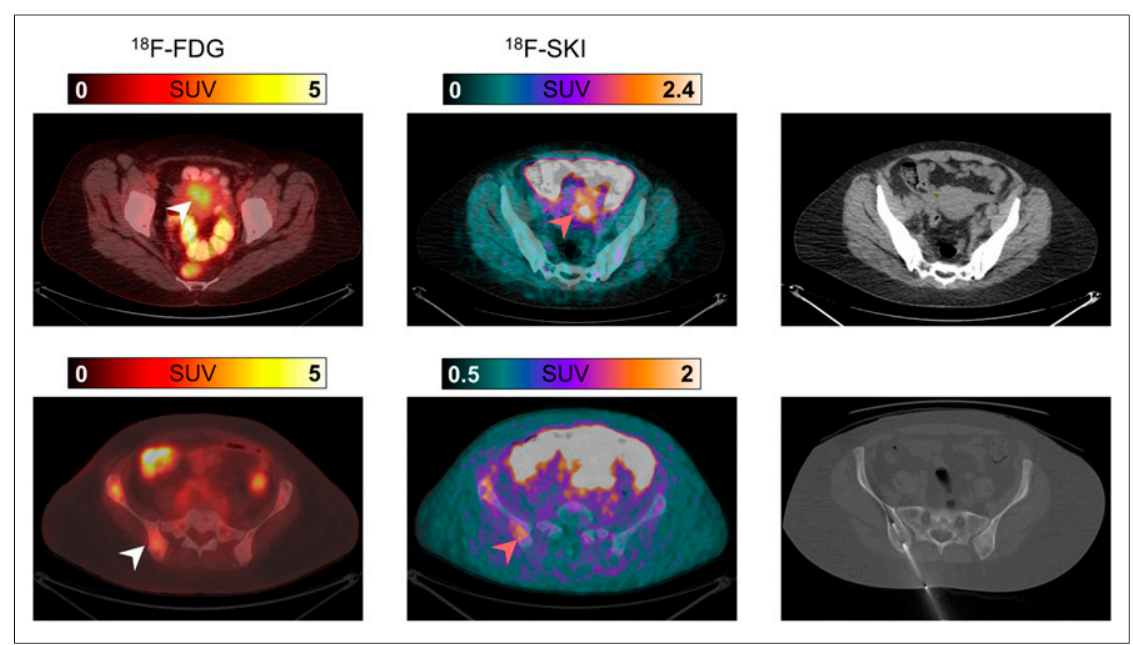

FIGURE 3. Biopsy-proven uterine and right iliac bone breast cancer metastases visualized on ${ }^{18} \mathrm{~F}-\mathrm{FDG}$ and ${ }^{18} \mathrm{~F}-\mathrm{SKI}$ PET/CT (patient 2). Axial ${ }^{18} \mathrm{~F}-\mathrm{FDG}$ PET/CT at $60 \mathrm{~min}$ after injection shows focal uptake in uterus (top, white arrow). Corresponding uptake is seen on axial ${ }^{18} \mathrm{~F}-\mathrm{SKI}$ PET/CT image at $90 \mathrm{~min}$ after injection (top, orange arrow). On noncontrast companion CT image, no definite soft-tissue mass is noted. Additionally, focal uptake in right pelvic bone is noted on ${ }^{18} \mathrm{~F}$ FDG (bottom, white arrow) and ${ }^{18} \mathrm{~F}-\mathrm{SKI}$ PET/CT images (bottom, orange arrow). This lesion was histologically confirmed by image-guided biopsy.

muscle (patient 3, with renal cell cancer) (Fig. 4), neither of which was known at the time of imaging. Lesion uptake at 30 and $90 \mathrm{~min}$ remained relatively unchanged, with a mean $\mathrm{SUV}_{\text {peak }}$ of $1.4 \pm 0.6$ (range, 0.6-3.2) and $1.4 \pm 0.4$ (range, 0.8-2.5), respectively. In the $2 \mathrm{MBC}$ patients with multiple predominant bone lesions and the patient with renal cell cancer, who had consecutive wholebody sweeps immediately after injection, lesion uptake was rapid (Fig. 2), typically plateauing by $30 \mathrm{~min}$ after injection and remaining close to the plateau in about $50 \%$ of lesions thereafter. About $30 \%$ of lesions demonstrated a continued slow increase in uptake. Few lesions (20\%) showed a slow washout after 30 min.

To determine the intralesional concentration of the radiotracer, 4 lesions were selected as reference lesions. Lesion sites included skeletal muscle (patient 3), uterus (patient 2), and bone (2 lesions
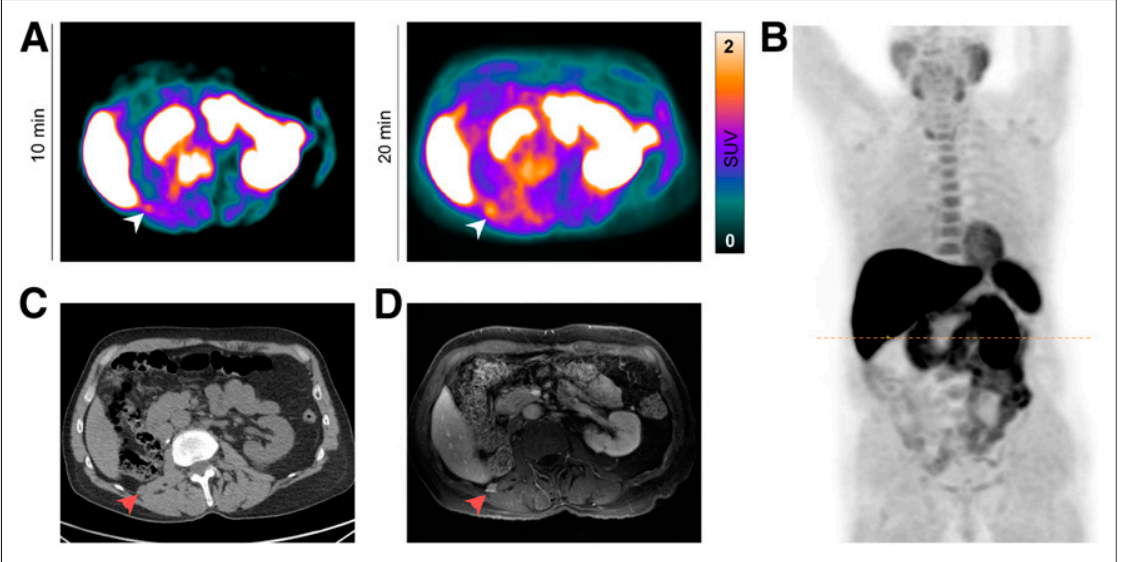

FIGURE 4. Focal ${ }^{18} \mathrm{~F}-\mathrm{SKI}$ uptake in right psoas muscle is consistent with metastasis (patient 3 ). (A) Axial PET images at 10 and 20 min demonstrate small focus of uptake in musculature (white arrow). (B) Maximum-intensity-projection image at 20 min shows prominent liver uptake; no definite lesion is seen. Dotted line indicates location of lesion. Of note, this was the only metastasis in this patient (status: after right nephrectomy). (C) Noncontrast companion CT image shows subtle low-attenuation area in right psoas muscle (white arrow). (D) This area shows contrast enhancement as seen on axial T1-weighted postcontrast MR image. in patient 1). Intratumoral drug concentrations ranged from 0.03 to $0.07 \mathrm{nM}$. In all reference lesions, a constant accumulation of tracer was observed 30-90 min after injection (Fig. 6).

\section{Absorbed Dose Estimates for ${ }^{18} \mathrm{~F}-\mathrm{SKI}$}

Normal-tissue and total-body time-activity curves are presented in Supplemental Figures 3 and 4, respectively, and residence times are presented in Supplemental Table 2. The full set of normal-tissue absorbed dose estimates $(\mathrm{mGy} / \mathrm{MBq})$ is shown in Table 2. The highest values (mean $\pm \mathrm{SD}$ ) were in the right colon $(0.167 \pm 0.04)$, small intestine $(0.153 \pm 0.03)$, and liver $(0.09 \pm 0.02)$. The effective dose was 0.0258 (SD, 0.0034) mSv/MBq.

\section{DISCUSSION}

This first-in-humans study of ${ }^{18} \mathrm{~F}-\mathrm{SKI}$ demonstrated the feasibility of noninvasive in vivo measurement and visualization of dasatinib-target engagement in metastatic solid and leukemic tumors. Tumor uptake was rapid and resulted in distinct lesion-to-background contrast. The lack of uptake in the brain may facilitate intracranial lesion detection, which is limited on ${ }^{18} \mathrm{~F}$-FDG PET by, for example, physiologic intense cortical uptake. ${ }^{18} \mathrm{~F}$-SKI also detected an unknown metastasis in the uterus in one of the patients with MBC who underwent ${ }^{18} \mathrm{~F}-\mathrm{FDG}$ PET 3 wk before ${ }^{18} \mathrm{~F}$-SKI. Although uterine ${ }^{18} \mathrm{~F}$-FDG uptake was observed, this was not further evaluated, as ${ }^{18} \mathrm{~F}-\mathrm{FDG}$ uptake can be physiologic, related to the menstrual cycle. In the patient with renal cell cancer, a subcentimeter-sized muscle lesion was newly diagnosed on the basis of ${ }^{18} \mathrm{~F}-\mathrm{SKI}$ uptake and confirmed on subsequent MRI. Despite the small sample size, these findings render ${ }^{18} \mathrm{~F}-\mathrm{SKI}$ an attractive companion diagnostic for disease assessment.

${ }^{18} \mathrm{~F}$-SKI PET imaging showed intense tracer uptake in the liver, gallbladder, and intestine related to the predominant hepatobiliary clearance, which impairs lesion detectability in the abdomen. Notable accumulation was also found in the spleen and kidney. The upper large intestines received the highest absorbed dose, consistent with its hepatobiliary excretion. Hepatobiliary excretion of ${ }^{18} \mathrm{~F}-\mathrm{SKI}$ is similar to that of dasatinib (18). ${ }^{18} \mathrm{~F}-\mathrm{SKI}-$ related radioactivity initially decreased rapidly in blood. After $30 \mathrm{~min}$, intact tracer accounted for $55 \%$ of the total radiation activity in plasma, suggesting that metabolites contributed significantly to the total radiation. ${ }^{18} \mathrm{~F}-\mathrm{SKI}$ is a lipophilic compound and is believed to enter cells by passive diffusion, similarly to dasatinib (20). The observed prolonged background clearance of ${ }^{18} \mathrm{~F}$ SKI is probably due to binding of the tracer or its metabolites to plasma proteins, as is the case with dasatinib (21). ${ }^{18} \mathrm{~F}-\mathrm{SKI}$ and dasatinib share nearly identical chemical 


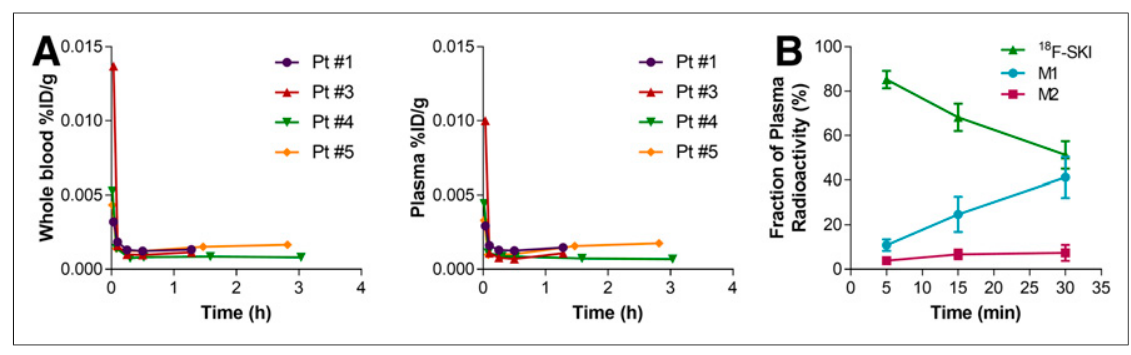

FIGURE 5. Whole-blood and plasma radioactivity of ${ }^{18} \mathrm{~F}-\mathrm{SKI}$. Initial rapid clearance of radioactivity from whole blood and plasma was observed in all 4 patients.

structures, and as such, their metabolites are expected to be similar. The dosimetry of ${ }^{18} \mathrm{~F}-\mathrm{SKI}$ evaluated in this study is in reasonable agreement with absorbed dose estimates previously derived by extrapolation from murine biodistribution studies (15).

We observed a gradual, progressive net accumulation of ${ }^{18} \mathrm{~F}$ SKI in tumors during the initial $30 \mathrm{~min}$ after tracer injection, followed by a plateau phase yielding distinct contrast to enable lesion visualization. Although most lesions (80\%) demonstrated this pattern of uptake or a continued rise in uptake, some $(20 \%)$ showed a slow washout after $30 \mathrm{~min}$. Further studies including dynamic scans are warranted to assess detailed uptake kinetics. In addition, to better characterize and ultimately elucidate the underlying biologic mechanism of the tumor signal, we are planning quantitative correlative analyses of lesion uptake and tissue expression of Src and other targets.

With a median administered dose of $212 \mathrm{MBq}$, as compared with common doses of $370 \mathrm{MBq}$ for ${ }^{18} \mathrm{~F}-\mathrm{FDG}$ PET, further dose escalation to optimize imaging parameters should be investigated in future clinical studies. Also, in view of the mass in the range of $0.3-4.1 \mu \mathrm{g}$ of precursor used in this study, which corresponds to more than a 24,000 -fold reduction from the equivalent therapeutic dose of dasatinib after standard dosing of $100 \mathrm{mg} / \mathrm{d}$, further increases in administered dose and corresponding mass are not expected to cause a therapeutic effect (11).

Early-phase clinical trials of dasatinib monotherapy have demonstrated that a significant minority of patients with cancer benefit from dasatinib after conventional therapy fails. To identify this important responder subset, new predictive biomarkers are urgently needed. Although multiple surrogate biomarkers for response to dasatinib have been described, such as measuring vascular epidermal growth factor receptor 2, plasma $\mathrm{N}$-telopeptide, and circulating tumor cells $(22,23)$ in blood, as well as imaging $\alpha_{v} \beta_{3}(24),{ }^{18} \mathrm{~F}-\mathrm{SKI}$ PET offers the first investigational clinical assay of in vivo tumor kinetics and engagement of tumor dasatinib targets with a dasatinib-derivative compound. The need for predictive biomarkers is illustrated in our clinical study (NCT00820170), in which dasatinib and paclitaxel combination therapy in molecularly unselected MBC patients failed to show a correlation between clinic-radiologic response and changes in the surrogate response biomarkers $\mathrm{N}$-terminal telopeptide, vascular epidermal growth factor, and circulating tumor cells (23). Of note, patients 1 and 2 were enrolled into this study after undergoing ${ }^{18} \mathrm{~F}$-SKI PET, and stable osseous disease in both patients was observed.

Using ${ }^{18} \mathrm{~F}-\mathrm{SKI}$ as a radiotracer to estimate therapeutic dosing requirements, we calculated intratumoral drug concentrations of $0.03-0.07 \mathrm{nM}$, approximately 1,000-fold less than pharmacologically relevant plasma concentrations. Surrogate imaging tools for receptor-targeted therapeutics, such as ${ }^{18} \mathrm{~F}$-fluorodihydrotestosterone for MDV3100 (enzalutamide), demonstrated clinical utility in understanding tumor biology and target receptor occupancy (25). Accordingly, the role of ${ }^{18} \mathrm{~F}-\mathrm{SKI}$ PET in dose selection, assessment of lesion-specific drug exposure, and biologic readout of kinase expression status should be assessed in future trials involving dasatinib.

TABLE 2

Absorbed Doses to Normal Tissues from ${ }^{18} \mathrm{~F}-\mathrm{SKI}(n=5)$

\begin{tabular}{|c|c|c|}
\hline \multirow[b]{2}{*}{ Target region } & \multicolumn{2}{|c|}{$\begin{array}{l}\text { Absorbed dose } \\
\text { (mGy/MBq) }\end{array}$} \\
\hline & Mean & SD \\
\hline Adrenals & 0.0270 & 0.00356 \\
\hline Brain & 0.00636 & 0.00291 \\
\hline Breasts & 0.00513 & 0.00127 \\
\hline Esophagus & 0.0112 & 0.000534 \\
\hline Eyes & 0.00440 & 0.00163 \\
\hline Gallbladder wall & 0.0327 & 0.00855 \\
\hline Left colon & 0.0575 & 0.0176 \\
\hline Small intestine & 0.153 & 0.0278 \\
\hline Stomach wall & 0.0131 & 0.00102 \\
\hline Right colon & 0.167 & 0.0402 \\
\hline Rectum & 0.0155 & 0.00373 \\
\hline Heart wall & 0.0126 & 0.00120 \\
\hline Kidneys & 0.0461 & 0.00337 \\
\hline Liver & 0.0904 & 0.0200 \\
\hline Lungs & 0.0111 & 0.000712 \\
\hline Ovaries & 0.0125 & 0.000903 \\
\hline Pancreas & 0.0255 & 0.00231 \\
\hline Prostate & 0.00678 & \\
\hline Salivary glands & 0.00916 & 0.00162 \\
\hline Red marrow & 0.0204 & 0.00460 \\
\hline Osteogenic cells & 0.0395 & 0.0114 \\
\hline Spleen & 0.0406 & 0.00918 \\
\hline Testes & 0.00129 & \\
\hline Thymus & 0.00630 & 0.00150 \\
\hline Thyroid & 0.00474 & 0.00164 \\
\hline Urinary bladder wall & 0.00709 & 0.00148 \\
\hline Uterus & 0.0137 & 0.000755 \\
\hline Total Body & 0.0130 & 0.00178 \\
\hline Effective dose (mSv/MBq) & 0.0258 & 0.00340 \\
\hline
\end{tabular}




\section{CONCLUSION}

Our study demonstrated that ${ }^{18} \mathrm{~F}$-SKI PET represents a promising clinical assay for imaging in vivo tumor targeting by a dasatinib-derivative compound. Our first-in-humans data support the continued clinical development of ${ }^{18} \mathrm{~F}-\mathrm{SKI}$.

\section{DISCLOSURE}

This work was supported by NIH/NCI grants R21 CA167803, P50 CA086438, and R01 CA164490. Memorial Sloan Kettering core facilities are supported by NIH/NCI Cancer Center support grant P30 CA008748. Additional salary support was provided by NCI grants R01 CA172546 (to Mark Dunphy) and R21 CA191913 (to Darren Veach). Simone Krebs was supported in part by the NIH/NCI Paul Calabresi Career Development Award for Clinical Oncology (K12 CA184746). Lukas Carter acknowledges support from the Ruth L. Kirschstein National Research Service Award postdoctoral fellowship (F32-EB025050). No other potential conflict of interest relevant to this article was reported.

\section{ACKNOWLEDGMENT}

We thank Leah Bassity for editing the manuscript.

\section{KEY POINTS}

QUESTION: What is the feasibility of noninvasive in vivo Src kinase-targeted tumor detection using ${ }^{18} \mathrm{~F}-\mathrm{SKI}$ for PET imaging?

PERTINENT FINDINGS: Five patients with a prior diagnosis of breast cancer, renal cell cancer, or leukemia were included as part of a prospective study. PET images showed multiple osseous metastases in the 2 patients with breast cancer. Furthermore, previously undiagnosed metastases in uterus and muscle from primary breast tumor and renal cell carcinoma, respectively, were visualized. Using ${ }^{18} \mathrm{~F}-\mathrm{SKI}$ as a quantitative molecular imaging tool to interrogate dosing requirements, we calculated intratumoral drug concentrations (range, 0.03-0.07 nM).

IMPLICATIONS FOR PATIENT CARE: ${ }^{18} \mathrm{~F}-\mathrm{SKI}$ offers potential as a pharmacometric biomarker to guide dasatinib-based cancer therapies, thus increasing the scope of personalized medicine.

\section{REFERENCES}

1. Hanahan D, Weinberg RA. The hallmarks of cancer. Cell. 2000;100:57-70.

2. Hanahan D, Weinberg RA. Hallmarks of cancer: the next generation. Cell. 2011;144: 646-674.

3. Summy JM, Gallick GE. Src family kinases in tumor progression and metastasis. Cancer Metastasis Rev. 2003;22:337-358.

4. Felsenfeld DP, Schwartzberg PL, Venegas A, Tse R, Sheetz MP. Selective regulation of integrin-cytoskeleton interactions by the tyrosine kinase Src. Nat Cell Biol. 1999;1:200-206.
5. Parsons SJ, Parsons JT. Src family kinases, key regulators of signal transduction. Oncogene. 2004;23:7906-7909.

6. Talpaz M, Shah NP, Kantarjian H, et al. Dasatinib in imatinib-resistant Philadelphia chromosome-positive leukemias. N Engl J Med. 2006;354:2531-2541.

7. Reissig D, Clement J, Sanger J, Berndt A, Kosmehl H, Bohmer FD. Elevated activity and expression of Src-family kinases in human breast carcinoma tissue versus matched non-tumor tissue. J Cancer Res Clin Oncol. 2001;127:226-230.

8. Sun J, Wang X, Tang B, et al. A tightly controlled Src-YAP signaling axis determines therapeutic response to dasatinib in renal cell carcinoma. Theranostics. 2018;8:3256-3267.

9. Lombardo LJ, Lee FY, Chen P, et al. Discovery of N-(2-chloro-6-methyl-phenyl)2-(6-(4-(2-hydroxyethyl)-piperazin-1-yl)-2-methylpyrimidin-4-ylamino)thiazole5-carboxamide (BMS-354825), a dual Src/Abl kinase inhibitor with potent antitumor activity in preclinical assays. J Med Chem. 2004;47:6658-6661.

10. Araujo J, Logothetis C. Dasatinib: a potent SRC inhibitor in clinical development for the treatment of solid tumors. Cancer Treat Rev. 2010;36:492-500.

11. Demetri GD, Lo Russo P, MacPherson IR, et al. Phase I dose-escalation and pharmacokinetic study of dasatinib in patients with advanced solid tumors. Clin Cancer Res. 2009;15:6232-6240.

12. Strand J, Varasteh Z, Eriksson O, Abrahmsen L, Orlova A, Tolmachev V. Gallium-68-labeled affibody molecule for PET imaging of PDGFR $\beta$ expression in vivo. Mol Pharm. 2014;11:3957-3964.

13. Wagner M, Wuest M, Hamann I, Lopez-Campistrous A, McMullen TPW, Wuest F. Molecular imaging of platelet-derived growth factor receptor-alpha (PDGFR $\alpha$ ) in papillary thyroid cancer using immuno-PET. Nucl Med Biol. 2018;58: 51-58.

14. Veach DR, Namavari M, Pillarsetty N, et al. Synthesis and biological evaluation of a fluorine-18 derivative of dasatinib. J Med Chem. 2007;50:5853-5857.

15. Dunphy MP, Zanzonico P, Veach D, et al. Dosimetry of ${ }^{18} \mathrm{~F}$-labeled tyrosine kinase inhibitor SKI-249380, a dasatinib-tracer for PET imaging. Mol Imaging Biol. 2012;14:25-31.

16. Li X, He Y, Ruiz CH, Koenig M, Cameron MD, Vojkovsky T. Characterization of dasatinib and its structural analogs as CYP3A4 mechanism-based inactivators and the proposed bioactivation pathways. Drug Metab Dispos. 2009;37:1242-1250.

17. Benezra M, Hambardzumyan D, Penate-Medina O, et al. Fluorine-labeled dasatinib nanoformulations as targeted molecular imaging probes in a PDGFB-driven murine glioblastoma model. Neoplasia. 2012;14:1132-1143.

18. Christopher LJ, Cui D, Wu C, et al. Metabolism and disposition of dasatinib after oral administration to humans. Drug Metab Dispos. 2008;36:1357-1364.

19. Stabin MG, Sparks RB, Crowe E. OLINDA/EXM: the second-generation personal computer software for internal dose assessment in nuclear medicine. $\mathrm{J} \mathrm{Nucl}$ Med. 2005;46:1023-1027.

20. Hiwase DK, Saunders V, Hewett D, et al. Dasatinib cellular uptake and efflux in chronic myeloid leukemia cells: therapeutic implications. Clin Cancer Res. 2008; 14:3881-3888

21. Luo FR, Yang Z, Camuso A, et al. Dasatinib (BMS-354825) pharmacokinetics and pharmacodynamic biomarkers in animal models predict optimal clinical exposure. Clin Cancer Res. 2006;12:7180-7186.

22. Somlo G, Atzori F, Strauss LC, et al. Dasatinib plus capecitabine for advanced breast cancer: safety and efficacy in phase I study CA180004. Clin Cancer Res. 2013;19:1884-1893.

23. Morris PG, Rota S, Cadoo K, et al. Phase II study of paclitaxel and dasatinib in metastatic breast cancer. Clin Breast Cancer. 2018;18:387-394.

24. Dumont RA, Hildebrandt I, Su H, et al. Noninvasive imaging of alphaVbeta3 function as a predictor of the antimigratory and antiproliferative effects of dasatinib. Cancer Res. 2009;69:3173-3179.

25. Scher HI, Beer TM, Higano CS, et al. Antitumour activity of MDV3100 in castration-resistant prostate cancer: a phase 1-2 study. Lancet. 2010;375:14371446 . 